\title{
Co-administration of prostaglandin E1 with somatostatin attenuates acute liver damage after massive hepatectomy in rats via inhibition of inflammatory responses, apoptosis and endoplasmic reticulum stress
}

\author{
CHANGJUN JIA, CHAOLIU DAI, XIANMIN BU, SONGLIN PENG, FENG XU, \\ YONGQING XU and YANG ZHAO
}

\begin{abstract}
Department of Hepato-Biliary-Splenic Surgery, Shengjing Hospital, China Medical University, Shenyang, Liaoning 110004, P.R. China
\end{abstract}

Received September 25, 2012; Accepted November 16, 2012

DOI: $10.3892 /$ ijmm.2012.1213

\begin{abstract}
Acute liver damage is considered to be the major cause of mortality after massive hepatectomy. Prostaglandin E1 (PGE1) and somatostatin (SST) have been shown to protect against hepatic injury of rats after partial hepatectomy. However, the precise mechanisms remain largely unknown. In this study, we examined the effects of PGE1, SST and the combination of these two drugs on acute liver damage of rats after $90 \%$ hepatectomy. We found that animal survival was improved when pretreated with PGE1 and SST. Portal venous pressure (PVP), serum alanine aminotransferase (ALT) and aspartate aminotransaminase (AST), tumor necrosis factor- $\alpha$ (TNF- $\alpha$ ) and interleukin-6 (IL-6) were all reduced after administration of PGE1 and SST. In addition, apoptosis was inhibited via upregulation of Bcl-2 and downregulation of Bax and caspase- 3 in drug treatment groups. Furthermore, pretreatment with PGE1 and SST alleviated endoplasmic reticulum (ER) stress by induction of heat shock protein 70 (HSP70) and glucose-regulated protein 78 (GRP78), but suppression of transcription factor $\mathrm{C} / \mathrm{EBP}$ homologous protein (CHOP). Our data suggest that administration of PGE1 and SST, particularly in combination, may prevent acute liver damage of rats after massive hepatectomy by inhibiting inflammatory responses, apoptosis and ER stress.
\end{abstract}

\section{Introduction}

Liver cancer, particularly primary liver cancer, is the leading cause of cancer-related mortality in both men and women in China $(1,2)$. At present, hepatectomy is recognized as the

Correspondence to: Dr Chaoliu Dai, Department of Hepato-BiliarySplenic Surgery, Shengjing Hospital, China Medical University, 36 Sanhao Street, Shenyang, Liaoning 110004, P.R. China

E-mail: chaoliu_dai@163.com

Key words: prostaglandin E1, somatostatin, liver failure, hepatectomy, apoptosis, endoplasmic reticulum stress first-line therapeutic method for primary and metastatic liver tumors (3). However, liver resection is restricted by the need to preserve a sufficient amount of hepatocytes to maintain normal physiological function $(4,5)$. Owing to improved understanding of the segmental anatomy of the liver and developments in surgical techniques, the mortality rate after liver resection has been reduced to $0-5 \%(6,7)$. Postresection liver failure (PLF), which is considered to be caused by excess inflammatory cytokines, reactive oxygen intermediates, apoptosis and disturbances of hepatic microcirculation, is the major cause of mortality following massive hepatectomy (8-11). Therefore, development of agents to prevent acute liver damage, particularly PLF, following liver resection, is of great significance in clinical application.

Prostaglandin E1 (PGE1) exerts a broad range of effects on cytoprotection and is widely used as a vasodilator in clinical practice (12). It has been shown that administration of liposome PGE1 (Lipo-PGE1) in rats could suppress ischemia-reperfusion (I/R) injury of the liver by improving microcirculation, reducing portal venous pressure (PVP) and alleviating parenchyma injury (13). Matsuo et al (14) reported that PGE1 induces heat shock proteins (HSPs) immediately after hepatic I/R. The intraportal infusion of PGE1 prevents the accumulation of excessive ascites and hyperbilirubinemia in patients with small-for-size graft (15). Moreover, it has been demonstrated that pretreatment of rats with PGE1 could improve the survival rate after massive hepatectomy by inhibiting apoptosis and stimulating hepatocyte proliferation as well as heat shock protein 70 (HSP70) accumulation (16-19). However, the underlying molecular mechanisms by which PGE1 exerts its protective effects on rats after massive hepatectomy are not fully elucidated. Therefore, a better understanding of such mechanisms will greatly promote the application of PGE1 to prevent acute liver damage after liver resection in the clinical setting.

Somatostatin (SST), a regulatory peptide hormone, is widely distributed in the body and is involved in multiple biological processes $(20,21)$. Previous reports have documented that SST was able to suppress hepatic I/R injury (22) 
and reduce PVP (23). Additionally, it has been shown that administration of SST or its analogues, octreotide, could exert protective effects against hepatic injury of rats undergoing partial hepatectomy and liver transplantation (24-28). However, it remains unknown whether SST possesses similar effects on rats undergoing massive hepatectomy.

In the present study, we used $90 \%$ hepatectomy of rats as a model to investigate whether SST can potentiate the therapeutical effects of PGE1 on rats undergoing massive liver resection. Our results showed that co-administration of PGE1 with SST attenuated acute liver damage after massive hepatectomy in rats more efficiently than either drug alone. The underlying mechanisms are associated with inhibition of inflammatory responses, apoptosis and endoplasmic reticulum (ER) stress.

\section{Materials and methods}

Animals and drugs. Male Sprague-Dawley rats, weighing 250-350 g, were purchased from the Experimental Animal Center of Shengjing Hospital. They were housed in an airconditioned room at $25^{\circ} \mathrm{C}$ with a controlled $12 \mathrm{~h}$ dark/light cycle. The animals received human care with unlimited access to chow and water. The surgeries were performed between 9 a.m. and 5 p.m.. All rats were fasted for $12 \mathrm{~h}$ before surgery. The animal study protocol was reviewed and approved by the Ethics Committee of China Medical University. SST (100 $\mu \mathrm{g} / \mathrm{vial})$ was obtained from Sandoz (Basel, Switzerland) and dissolved in physiological saline. PGE1 $(10 \mu \mathrm{g} / \mathrm{vial})$ was purchased from Tide Pharmaceutical Co., Ltd. (Beijing, China). One hundred micrograms PGE1 were dissolved in $20 \mathrm{ml}$ physiological saline. A dose of $0.5 \mu \mathrm{g} / \mathrm{kg} / \mathrm{min}$ PGE1 and $20 \mu \mathrm{g} / \mathrm{kg}$ body weight SST were used in the present study.

Experimental protocol. All rats were randomly divided into the control, SST, PGE1 or combined treatment group, with 26 rats each group. Rats in the SST group were injected intravenously with SST $(20 \mu \mathrm{g} / \mathrm{kg}$ body weight, dissolved in $1 \mathrm{ml}$ physiological saline) $30 \mathrm{~min}$ before the operation. During the surgery, physiological saline was continuously pumped intravenously and the total volume was the same as PGE1 treatment. Another intravenous injection of SST ( $20 \mu \mathrm{g} / \mathrm{kg}$ body weight) was given after the hepatectomy. Rats in the PGE1 group received $1 \mathrm{ml}$ physiological saline $30 \mathrm{~min}$ before the operation and continuous intravenous pumping $(0.5 \mu \mathrm{g} / \mathrm{kg} / \mathrm{min})$ during the surgery. Instead of SST, $1 \mathrm{ml}$ physiological saline was given after the operation. For the combined group, the treatment protocol was the same as the PGE1 group, except that SST (20 $\mu \mathrm{g} / \mathrm{kg}$ body weight) was injected before or after the operation instead of physiological saline alone. Rats in the control group received physiological saline during the entire process. All animals were anesthetized with sodium pentobarbital (40 mg/kg) and the surgery was performed as described by Togo et al (18). Briefly, the rats were placed in a supine position and then a midline incision was made. After detaching from surrounding tissues, the liver was pushed out. All rats received a hypodermic injection of $10 \%$ glucose after the hepatectomy. Animals were sacrificed (16 rats in each group) at $10 \mathrm{~min}, 6$, 12 and $24 \mathrm{~h}$ after the operation, except for survival observation (10 rats in each group). Liver specimens and blood samples were collected at each time point. The residual livers were fixed in $10 \%$ formalin or frozen in liquid nitrogen immediately, and were then subjected to histological examination or western blot analysis. PVP was measured by presser transducer which was placed into the portal vein after puncturing the vein with a 23-gauge needle. Activities of ALT and AST in serum were determined by the automatic Biochemistry Analyzer (Abbott Laboratories, Irving, TX, USA).

Histological examination. The remnant liver tissues were collected at $10 \mathrm{~min}, 6,12$ and $24 \mathrm{~h}$ after $90 \%$ hepatectomy for all groups. Liver samples were fixed in $10 \%$ neutral buffered formalin and then frozen section was performed. Subsequently, these sections were stained with routine hematoxylin and eosin (H\&E) to evaluate the morphological alterations by using a standard protocol.

Enzyme-linked immunosorbent assay (ELISA). ELISA kits (Senxiong Biotechnology Co., Ltd., Shanghai, China) were used to measure the concentration of tumor necrosis factor- $\alpha$ (TNF- $\alpha$ ) and interleukin-6 (IL-6) in serum according to the manufacturer's instructions. The sample concentrations were calculated from a standard curve and expressed as $\mathrm{pg} / \mathrm{ml}$.

Western blot analysis. Liver tissues were obtained at $10 \mathrm{~min}$, 6,12 and $24 \mathrm{~h}$ after $90 \%$ hepatectomy from all groups. The samples were weighed, sectioned and then washed with $0.9 \%$ $\mathrm{NaCl}$. The homogenates were sonicated for $5 \mathrm{sec}, 20-30$ times, and then centrifuged at $12,000 \mathrm{x}$ g for $30 \mathrm{~min}$ at $4^{\circ} \mathrm{C}$. Following centrifugation, supernatant was collected and the protein concentrations were determined using a bovine serum albumin (BSA) standard line. Equal amounts of protein were separated on sodium dodecyl sulfate polyacrylamide gel electrophoresis (SDS-PAGE) and then transferred electrophoretically onto polyvinylidene difluoride (PVDF) membranes (Millipore, Bedford, MA, USA). The blotted membranes were blocked with $5 \%$ non-fat dry milk (w/v) in Tris-buffered saline with $0.1 \%$ Tween-20 (TBST). Subsequently, the membranes were incubated with anti-TNF- $\alpha$, IL-6, Bax, Bcl-2, caspase-3, HSP70, glucose-regulated protein 78 (GRP78) and CHOP antibodies, respectively (1:1,000 dilution; Santa Cruz Biotechnology, Inc., Santa Cruz, CA, USA) overnight at $4^{\circ} \mathrm{C}$. After three rinses with TBST at 10-min intervals, the membranes were incubated with horseradish peroxidase-labeled goat anti-rabbit IgG (1:5,000 dilution; Santa Cruz Biotechnology, Inc.,) for $2 \mathrm{~h}$ at room temperature. Then, protein bands were visualized by an ECL plus chemiluminescence kit (Millipore). Glyceraldehyde3 -phosphate dehydrogenase (GAPDH) was used as a loading control.

Terminal deoxynucleotidyl transferase dUTP nick-end labeling (TUNEL) staining. Apoptosis was evaluated by the TUNEL staining which was performed with a commercially available in situ cell death detection kit (ZSGB-Bio Co., Ltd., Beijing, China), according to the manufacturer's instructions. Liver samples were embedded in paraffin and then sectioned at a thickness of $4 \mu \mathrm{m}$. The sections were dewaxed in xylene and rehydrated through a graded series of ethanol (100-70\%). Then, the sections were digested with proteinase $\mathrm{K}(40 \mu \mathrm{g} / \mathrm{ml})$ for $10 \mathrm{~min}$ at $37^{\circ} \mathrm{C}$. After digestion, the sections were incubated 


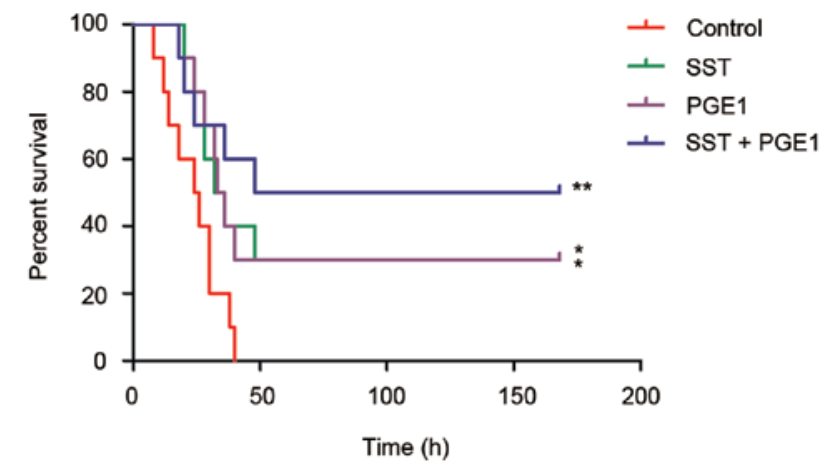

Figure 1. Effect of PGE1 and SST on survival after 90\% hepatectomy. Rats were divided into the control, PGE1, SST or combined treatment group $(\mathrm{n}=10$, respectively). Survival was recorded until $168 \mathrm{~h}$ after hepatectomy. ${ }^{*} \mathrm{P}<0.05,{ }^{* *} \mathrm{P}<0.01$ by log-rank test.

with $3 \% \mathrm{H}_{2} \mathrm{O}_{2}$ for 10 min at room temperature to block the endogenous peroxidase activity. The slides were covered with citric acid buffer for $5 \mathrm{~min}$ and then with working solution which contained TdT enzyme overnight at $4^{\circ} \mathrm{C}$. After washing with PBS, the slides were incubated with peroxidase for $1 \mathrm{~h}$ at $37^{\circ} \mathrm{C}$. The color was developed with the DAB substrate kit and the reactions were terminated by tap water. The sections were then counterstained with hematoxylin, dehydrated and mounted. Total hepatocytes and apoptotic cells were counted from 5 random high-power fields for each section. Apoptotic index (apoptotic cells/100 hepatocytes) was used to evaluate the effect of PGE1 and SST on apoptosis after 90\% hepatectomy.

Statistical analysis. All data analyses were performed with SPSS 17.0 software package (SPSS Inc., Chicago, IL, USA) and are presented as the means \pm standard deviation (SD). Statistical analysis was evaluated by one-way ANOVA followed by LSD test unless otherwise stated. Survival rates after $90 \%$ hepatectomy were expressed using the Kaplan-Meier curves. The log-rank test was used for the survival statistics. $\mathrm{P}<0.05$ was considered to indicate a statistically significant difference.

\section{Results}

Co-administration of PGE1 with SST improves survival in rats after massive hepatectomy. To investigate the protective effects of PGE1 and SST on rats after massive hepatectomy, rats were treated as described in Materials and methods, and the survival of these rats was recorded for 7 days after $90 \%$ hepatectomy. The Kaplan-Meier method was used to analyze the obtained results. We found that both PGE1 and SST ameliorated the survival rate of rats after $90 \%$ hepatectomy compared with the control group $(\mathrm{P}<0.05$, log-rank test). The survival rate was improved significantly by co-administration of these two drugs $(\mathrm{P}<0.01$, log-rank test) (Fig. 1). Our data suggest that co-administration of PGE1 with SST exerts a much stronger protective effect on rats following massive hepatectomy than either drug alone.

Co-administration of PGEl with SST attenuates liver injury in rats following massive hepatectomy. Previous reports have
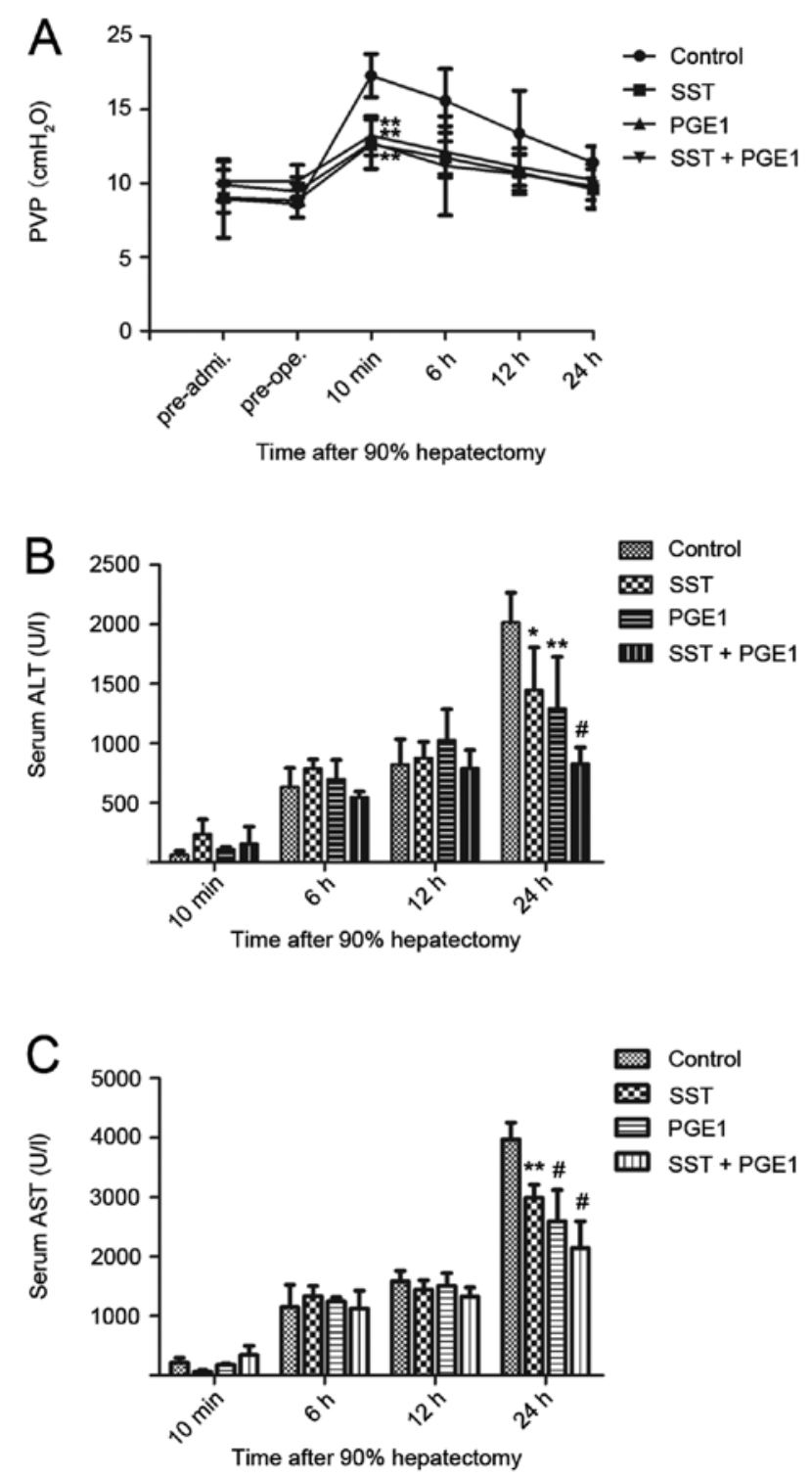

Figure 2. Effect of PGE1 and SST on PVP, ALT and AST levels after 90\% hepatectomy. (A) PVP was measured before administration and operation or $10 \mathrm{~min}, 6,12$ and $24 \mathrm{~h}$ after hepatectomy. (B) Serum ALT and (C) AST levels were measured $10 \mathrm{~min}, 6,12$ and $24 \mathrm{~h}$ after hepatectomy. Values are means $\pm \mathrm{SD}, \mathrm{n}=4 .{ }^{*} \mathrm{P}<0.05,{ }^{* *} \mathrm{P}<0.01,{ }^{*} \mathrm{P}<0.001$ vs. the control group.

shown that acute portal hypertension after hepatectomy could induce liver injury $(29,30)$. To determine whether PGE1 and SST can attenuate liver injury caused by acute portal hypertension in rats after massive hepatectomy, PVP was measured at different time points. As shown in Fig. 2A, rapid increases in PVP were observed 10 min after $90 \%$ hepatectomy in rats in all treatment groups. However, pretreatment with PGE1 and SST, either alone or in combination, significantly inhibited the increase in PVP compared to the control group $(\mathrm{P}<0.01)$.

Next, we analyzed the releases of alanine aminotransferase (ALT) and aspartate aminotransaminase (AST) in serum which was used as an indicator of liver injury. The obtained results showed that the serum levels of ALT and AST were elevated postoperatively in all groups. In contrast to the control group, co-administration of PGE1 with SST significantly reduced ALT and AST in serum $(\mathrm{P}<0.001)$ (Fig. 2B and C). H\&E staining results showed that no obvious 

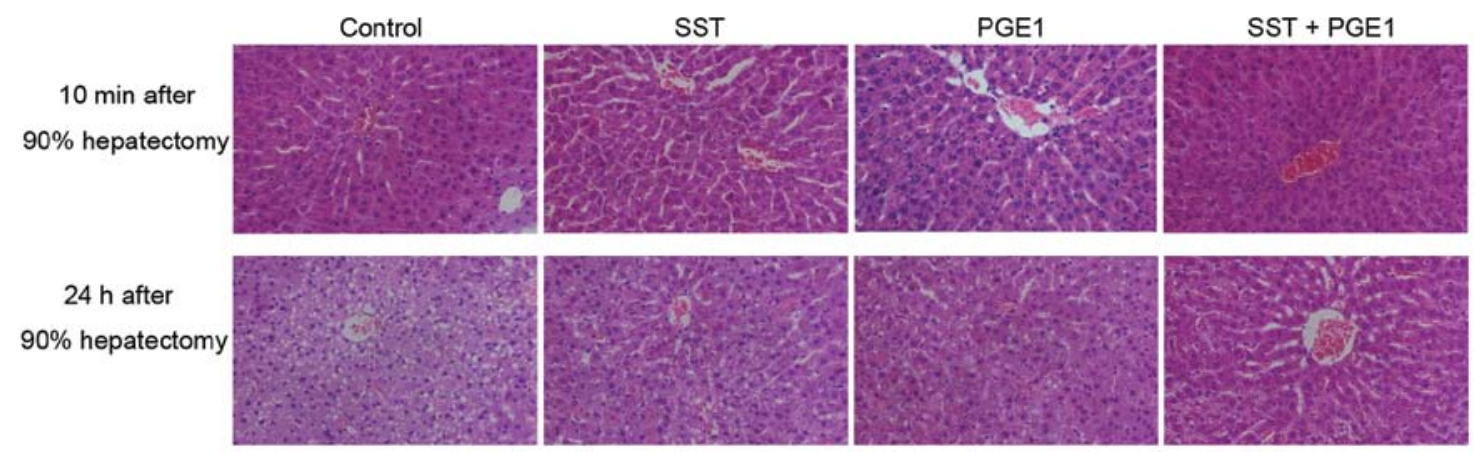

Figure 3. H\&E staining of the remnant liver after $90 \%$ hepatectomy. Histological alterations were observed $10 \mathrm{~min}$ or $24 \mathrm{~h}$ after hepatectomy for the control, PGE1, SST and combined treatment groups.
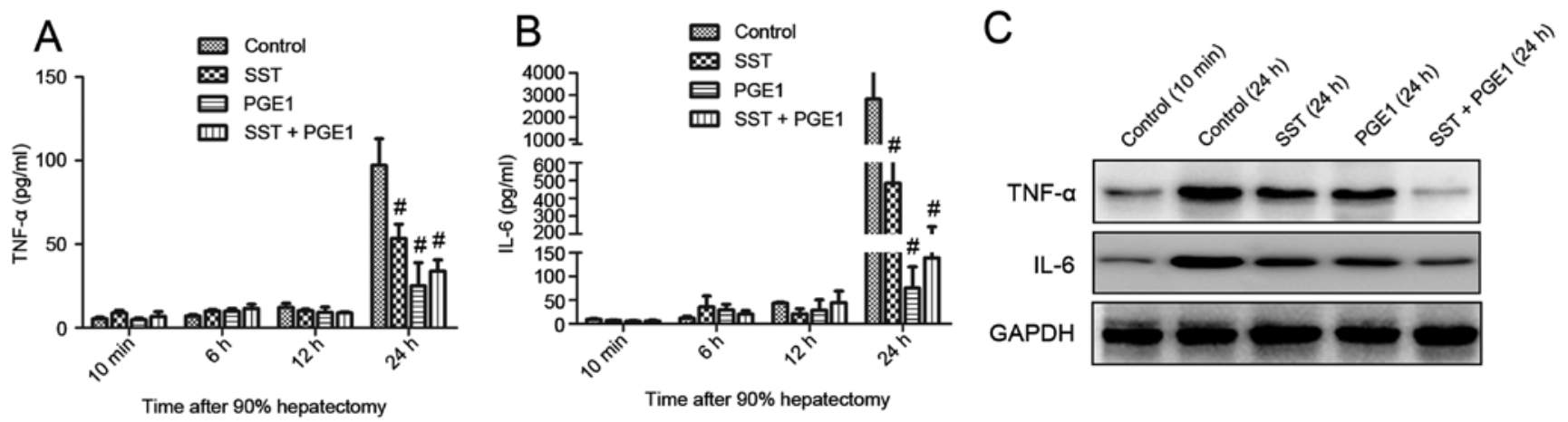

Figure 4. Effects of PGE1 and SST on the accumulation of inflammatory cytokines after 90\% hepatectomy. Levels of (A) TNF- $\alpha$ and (B) IL-6 in serum or (C) the remnant livers were measured 10 min, 6,12 and $24 \mathrm{~h}$ after hepatectomy for all groups. ${ }^{~} \mathrm{P}<0.001$ vs. the control group.

A

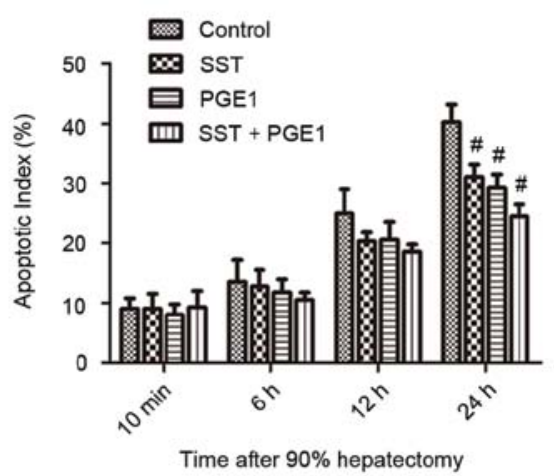

C

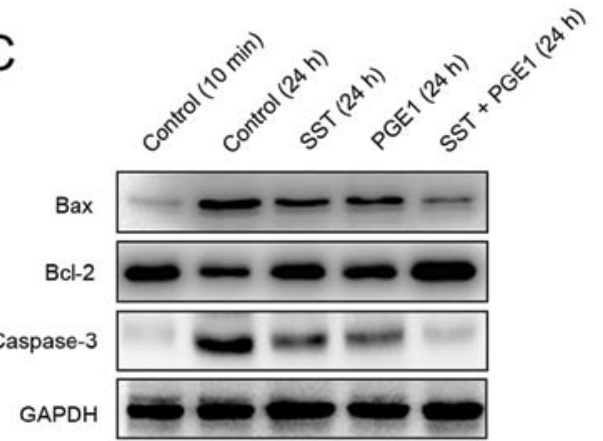

B

Control

SST

PGE1

SST + PGE1

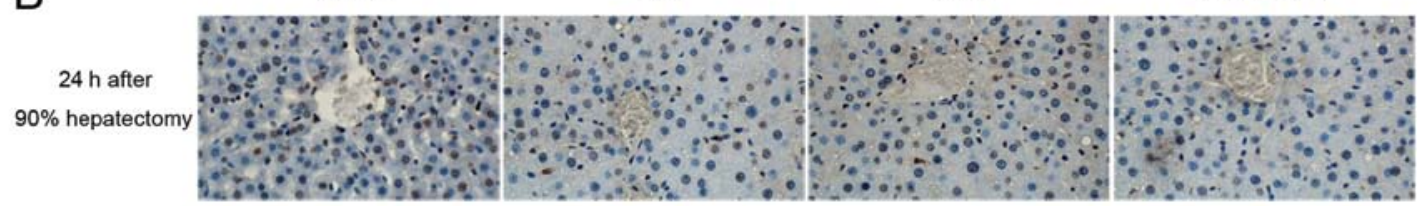

Figure 5. Effects of PGE1 and SST on apoptosis after 90\% hepatectomy. (A) The apoptotic index was determined 10 min, 6,12 and 24 h after hepatectomy by TUNEL assay. Values are the means $\pm \mathrm{SD}, \mathrm{n}=4 .{ }^{\#} \mathrm{P}<0.001 \mathrm{vs}$. the control group. (B) TUNEL staining results of the control, PGE1, SST and combined treatment groups $24 \mathrm{~h}$ after hepatectomy. (C) Effect of PGE1 and SST on the expression of apoptosis-related proteins. Western blotting results of Bax, Bcl-2, caspase-3, $10 \mathrm{~min}$ or $24 \mathrm{~h}$ after hepatectomy. Representative results of three independent experiments are shown. GAPDH was used as a loading control.

histological alterations were observed $10 \mathrm{~min}$ after $90 \%$ hepatectomy (Fig. 3). However, pathological changes, such as disorder structure of hepatic lobules, enlarged hepatic sinus, liver cell swelling and partial necrosis, appeared $24 \mathrm{~h}$ after massive hepatectomy in the control group (Fig. 3). Co-administration of PGE1 with SST abated these changes more efficiently than either PGE1 or SST alone (Fig. 3). Collectively, our data demonstrate that co-administration of 


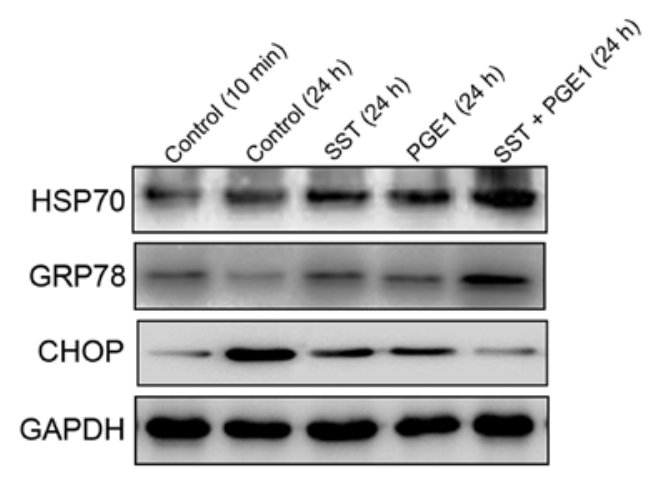

Figure 6. Effects of PGE1 and SST on ER stress. Western blotting results of UPR-related proteins $10 \mathrm{~min}$ or $24 \mathrm{~h}$ after hepatectomy. Representative results of three independent experiments are shown. GAPDH was used as a loading control.

PGE1 with SST attenuates liver injury in rats after massive hepatectomy.

Production of inflammatory cytokines is suppressed in rats after massive hepatectomy by co-administration of PGE1 with SST. Inflammatory cytokines, such as TNF- $\alpha$ and IL-6, have been shown to be involved in liver failure after massive resection $(31,32)$. Therefore, contents of TNF- $\alpha$ and IL-6 were determined in serum and the remnant liver. As shown in Fig. 4A and B, TNF- $\alpha$ and IL-6 were minimally expressed 10 min after $90 \%$ hepatectomy in serum from all groups. Twenty-four hours postoperatively, the levels of TNF- $\alpha$ and IL-6 were greatly increased in the control group. By contrast, these increases were significantly attenuated by administration of PGE1 and SST. Pretreatment with PGE1 alone was more effective than combined with SST in terms of inhibiting inflammatory cytokine accumulation in serum. We found similar results in remnant liver, except that co-administration of PGE1 with SST exhibited the most effective inhibition effect than either drug alone (Fig. 4C).

Co-administration of PGE1 with SST inhibits apoptosis in rats after massive hepatectomy. To investigate the effects of PGE1 and SST on apoptosis, TUNEL assays were performed. The results indicated that the apoptotic index (AI) increased over time for all groups. Twenty-four hours postoperatively, the AI value decreased from $40.25 \pm 2.99 \%$ for the control group to $29.25 \pm 2.21,31 \pm 2.16,24.5 \pm 2.08 \%$ for the SST, PGE1 and combined treatment groups, respectively (Fig. 5A). Co-administration of PGE1 with SST suppressed apoptosis more efficiently than either alone as evidenced by less TUNELpositive staining (Fig. 5B). Furthermore, we observed that pretreatment with PGE1 and SST downregulated expression levels of Bax and caspase- 3 and upregulated Bcl-2, particularly in the combined treatment group (Fig. 5C). These results indicate that co-administration of PGE1 with SST suppresses apoptosis in rats after liver resection.

Co-administration of PGE1 with SST alleviates ER stress in rats after massive hepatectomy. In order to eliminate ER stress, eukaryotic cells initiate unfolded protein response (UPR) which involves suspension of protein synthesis and upregulation of molecular chaperones. To explore whether PGE1 and SST alleviate ER stress, we analyzed the expression of UPR related proteins. Western blotting results showed that the expression levels of two molecular chaperones, HSP70 and GRP78, were significantly increased by co-administration of PGE1 with SST compared to the control group (Fig. 6). Transcription factor C/EBP homologous protein (CHOP), whose expression is upregulated by ER stress, is involved in ER stress-induced apoptosis (33). We found that pretreatment with PGE1 and SST significantly inhibited the expression of CHOP, particularly in the combined group (Fig. 6). These data suggest that co-administration of PGE1 with SST alleviates ER stress in the remnant liver.

\section{Discussion}

In the present study, we examined the protective effects of PGE1 and SST in rats after 90\% hepatectomy. Our data demonstrated that co-administration of PGE1 with SST attenuates liver injury and improves the survival rates in rats after massive hepatectomy more efficiently than either treatment alone. The underlying mechanisms include inhibition of inflammatory responses and apoptosis, and alleviation of ER stress.

Liver failure after massive hepatic resection is considered a major contributor to postoperative mortality (3). The development of liver failure involves several causes, such as excess inflammatory response, impaired liver regeneration and induction of apoptosis, abnormal hemodynamics, I/R injury and sepsis (34). Therefore, drug combinations which can exert multiple therapeutical actions are preferable. According to earlier findings, both PGE1 and SST have been shown to protect against liver injury produced by $\mathrm{I} / \mathrm{R}$ injury and partial hepatectomy $(13,16,22,25)$. The results obtained in the present study showed that application of PGE1 or SST significantly improved survival, reduced PVP, ALT, AST and alleviated the histological changes of rats after $90 \%$ hepatectomy. In particular, the combination of these two drugs exhibited a much stronger protective effect than either drug alone. It should also be noted that co-administration of PGE1 with SST did not show a significant difference in reducing PVP compared to PGE1 or SST alone. This may be due to the major effect of PGE1 on liver hemodynamics in increasing portal venous flow rather than reducing PVP (35).

Typically pro-inflammatory cytokines, such as TNF- $\alpha$ and IL-6, participate in the initiation of liver regeneration (36). A previous report showed that impaired liver regeneration after partial hepatectomy in ICAM-1 deficient mice is associated with a marked decrease in tissue TNF- $\alpha$ and IL- 6 levels. In addition, injection of recombinant IL- 6 could restore the proliferation of hepatocytes (37). Receptor for advanced glycation end products (RAGE) inhibits regeneration after massive liver injury by suppression of TNF- $\alpha$ (38). It has been shown that cold ischemia suppresses liver regeneration after partial liver transplantation in rats by decreasing the production of TNF- $\alpha$ and IL-6 in rats (39). However, excessive releases of these cytokines are tightly related to liver failure after massive hepatectomy. Ogata et al (32) reported that suppression of excessive TNF- $\alpha$ production using ONO-SM362, a TNF- $\alpha$ suppressant, ameliorates liver failure after $85 \%$ hepatectomy. Another study 
also showed that enhanced expressions of TNF- $\alpha$ are detected in rats after $85 \%$ hepatectomy which exhibit extensive patchy necrosis as compared with $70 \%$ hepatectomy (31). The results obtained in our present study are consistent with previous reports. We found that expressions of TNF- $\alpha$ and IL- 6 were significantly increased $24 \mathrm{~h}$ after $90 \%$ hepatectomy both in serum and the remnant liver. Furthermore, application of PGE1 and SST was able to downregulate the excessive expressions of TNF- $\alpha$ and IL-6 and thus alleviate liver injury. In addition, these cytokine levels were relatively higher even after administration of PGE1 and SST. This may be a benefit for maintaining and promoting regeneration of the remnant liver. However, the mechanisms animals use to maintain the balance of TNF- $\alpha$ and IL- 6 between liver injury and regeneration remain to be elucidated. Co-administration of PGE1 with SST inhibited the production of TNF- $\alpha$ and IL- 6 in the remnant liver more efficiently than in serum.

Apoptosis and necrosis of the remnant hepatocytes further aggravate liver injury and eventually result in liver failure following massive hepatectomy (8). Upregulation of caspases and Fas and downregulation of Bcl-2 have been found in rats which died of hepatic failure within $96 \mathrm{~h}$ after $95 \%$ hepatectomy (40). A previous study demonstrated that pretreatment with PGE1 significantly increases the expression levels of Bcl-xL in rats after $95 \%$ hepatectomy and thus improves the survival of these animals (16). Our results further confirmed the above findings. We observed that apoptosis increased significantly in rats $24 \mathrm{~h}$ after $90 \%$ hepatectomy. However, co-administration of PGE1 with SST reduced the occurrence of apoptosis markedly compared to the control group. Further analyses demonstrated that administration of PGE1 or SST in rats after $90 \%$ hepatectomy downregulated the expression levels of pro-apoptotic Bax and caspase-3, whereas it induced the accumulation of anti-apoptotic Bcl-2 and eventually inhibited apoptosis. Therefore, PGE1 and SST exert protective effects on rats after massive hepatectomy at least partially by inhibiting apoptosis.

The accumulation of misfolded proteins in the lumen of the ER results in ER stress. UPR, including suspension of protein synthesis, upregulation of molecular chaperones and accumulation of folding enzymes, is activated to eliminate such stress (41). ER stress has been observed among various liver diseases, such as chronic viral hepatitis B and C, I/R injury and alcoholinduced liver injury (42). However, the precise role of ER stress in rats after massive hepatectomy has yet to be demonstrated. HSP70 and GRP78 are two molecular chaperones that facilitate protein folding and thus eliminate ER stress. Enhanced expression of HSP70 has been found in rats after 95\% hepatectomy (18). However, sustained ER stress can lead to apoptosis (41). It has been shown that deletion of the transcription factor CHOP protects against ER stress-induced apoptosis (33). In the present study, we found that co-administration of PGE1 with SST greatly upregulated the protein expression levels of HSP70 and GRP78 and thus may contribute to alleviating the ER stress induced by hepatectomy. Moreover, CHOP was significantly inhibited when pretreated with the combination of PGE1 and SST. Therefore, co-administration of PGE1 with SST may exert a protective effect against rats after massive hepatectomy partially by inhibiting apoptosis induced by excessive ER stress.
In conclusion, our data demonstrated that co-administration of PGE1 with SST attenuated the acute liver damage of rats after $90 \%$ hepatectomy. The potential mechanisms included inhibition of inflammatory responses and apoptosis, and alleviation of ER stress induced by hepatectomy. Our findings have broad implications in promoting the application of the combination of these two drugs in clinical settings against liver failure after massive hepatectomy.

\section{References}

1. He J, Gu D, Wu X, et al: Major causes of death among men and women in China. N Engl J Med 353: 1124-1134, 2005.

2. Chen JG and Zhang SW: Liver cancer epidemic in China: past, present and future. Semin Cancer Biol 21: 59-69, 2011.

3. Hammond JS, Guha IN, Beckingham IJ and Lobo DN: Prediction, prevention and management of postresection liver failure. Br J Surg 98: 1188-1200, 2011.

4. Jarnagin WR, Gonen M, Fong Y, et al: Improvement in perioperative outcome after hepatic resection: analysis of 1,803 consecutive cases over the past decade. Ann Surg 236: 397-407, 2002.

5. Belghiti J, Hiramatsu K, Benoist S, Massault P, Sauvanet A and Farges O: Seven hundred forty-seven hepatectomies in the 1990s: an update to evaluate the actual risk of liver resection. J Am Coll Surg 191: 38-46, 2000.

6. Virani S, Michaelson JS, Hutter MM, et al: Morbidity and mortality after liver resection: results of the patient safety in surgery study. J Am Coll Surg 204: 1284-1292, 2007.

7. House MG, Ito H, Gonen M, et al: Survival after hepatic resection for metastatic colorectal cancer: trends in outcomes for 1,600 patients during two decades at a single institution. J Am Coll Surg 210: 744-755, 2010.

8. Hasegawa S, Kubota T, Fukuyama N, et al: Apoptosis of hepatocytes is a main cause of inducing lethal hepatic failure after excessive hepatectomy in rats. Transplant Proc 31: 558-559, 1999.

9. Mochida S, Ogata I, Hirata K, Ohta Y, Yamada S and Fujiwara K: Provocation of massive hepatic necrosis by endotoxin after partial hepatectomy in rats. Gastroenterology 99: 771-777, 1990.

10. Leist M, Gantner F, Bohlinger I, Tiegs G, Germann PG and Wendel A: Tumor necrosis factor-induced hepatocyte apoptosis precedes liver failure in experimental murine shock models. Am J Pathol 146: 1220-1234, 1995.

11. Andiran F, Ayhan A, Tanyel FC, Abbasoglu O and Sayek I: Regenerative capacities of normal and cirrhotic livers following $70 \%$ hepatectomy in rats and the effect of alpha-tocopherol on cirrhotic regeneration. J Surg Res 89: 184-188, 2000.

12. Kobayashi S, Baba H, Takeno K, et al: Blood flow analysis of compressed nerve root after intravenous injection of lipo-prostaglandin E1. J Orthop Res 27: 1252-1257, 2009.

13. Li JP, Wang Y, Huang YH, Li HP, Chen W and Yang JY: Experimental study of protective mechanism of Lipo-PGE(1) on hepatic ischemia-reperfusion injury. Zhonghua Yi Xue Za Zhi 89: 3371-3374, 2009 (In Chinese).

14. Matsuo K, Togo S, Sekido H, et al: Pharmacologic preconditioning effects: prostaglandin E1 induces heat-shock proteins immediately after ischemia/reperfusion of the mouse liver. J Gastrointest Surg 9: 758-768, 2005.

15. Suehiro T, Shimada M, Kishikawa K, et al: Effect of intraportal infusion to improve small for size graft injury in living donor adult liver transplantation. Transpl Int 18: 923-928, 2005.

16. Ishibe A, Togo S, Kumamoto T, et al: Prostaglandin E1 prevents liver failure after excessive hepatectomy in the rat by upregulating Cyclin C, Cyclin D1, and Bclxl. Wound Repair Regen 17: 62-70, 2009.

17. Ando K, Miyazaki M, Shimizu H, Okuno A and Nakajima N: Beneficial effects of prostaglandin $\mathrm{E}(1)$ incorporated in lipid microspheres on liver injury and regeneration after $90 \%$ partial hepatectomy in rats. Eur Surg Res 32: 155-161, 2000.

18. Togo S, Chen H, Takahashi T, et al: Prostaglandin E1 improves survival rate after 95\% hepatectomy in rats. J Surg Res 146: 66-72, 2008.

19. Yoshida N, Iwata H, Yamada T, et al: Improvement of the survival rate after rat massive hepatectomy due to the reduction of apoptosis by caspase inhibitor. J Gastroenterol Hepatol 22: 2015-2021, 2007. 
20. Dasgupta P: Somatostatin analogues: multiple roles in cellular proliferation, neoplasia, and angiogenesis. Pharmacol Ther 102: 61-85, 2004.

21. Pinter E, Helyes Z and Szolcsanyi J: Inhibitory effect of somatostatin on inflammation and nociception. Pharmacol Ther 112: 440-456, 2006.

22. Landa I, Arias J, Gomez M, Quadros M, Moreno A and Balibrea JL: Cytoprotective effect of somatostatin in a rat model of hepatic ischemic reperfusion. Hepatology 16: 1474-1476, 1992.

23. Villanueva $C$ and Balanzo J: Variceal bleeding: pharmacological treatment and prophylactic strategies. Drugs 68: 2303-2324, 2008.

24. Pruthi RS, Farouk M, Tsai WH, Michalopoulos G and Meyers WC: The effect of octreotide on hepatic regeneration in rats. Surgery 113: 84-89, 1993.

25. Yamamoto K, Takenaka K, Matsumata T, Shimada M and Sugimachi K: The effect of octreotide on morphological hepatic regeneration and hepatic functional recovery after a two-thirds hepatectomy in rats. Hepatogastroenterology 46: 1880-1884, 1999.

26. Hashimoto M, Kothary PC and Raper SE: The effects of transforming growth factor alpha and somatostatin on regenerating hepatocytes in the rat. Regul Pept 44: 49-59, 1993.

27. Feng ZY, Xu X, Wu LJ, Wu J, Zhu SM and Zheng SS Downregulation of endothelin-1 by somatostatin improves liver function of recipients undergoing adult-to-adult living donor liver transplantation. Chin Med J (Engl) 123: 1961-1966, 2010.

28. Xu X, Man K, Zheng SS, et al: Attenuation of acute phase shear stress by somatostatin improves small-for-size liver graft survival. Liver Transpl 12: 621-627, 2006.

29. Koyama S, Sato Y and Hatakeyama K: The subcutaneous splenic transposition prevents liver injury induced by excessive portal pressure after massive hepatectomy. Hepatogastroenterology 50 : 37-42, 2003.

30. Yachida S, Wakabayashi H, Kokudo Y, et al: Measurement of serum hyaluronate as a predictor of human liver failure after major hepatectomy. World J Surg 24: 359-364, 2000.
31. Panis Y, McMullan DM and Emond JC: Progressive necrosis after hepatectomy and the pathophysiology of liver failure after massive resection. Surgery 121: 142-149, 1997.

32. Ogata T, Yamashita K, Horiuchi H, Okuda K and Todo S: A novel tumor necrosis factor-alpha suppressant, ONO-SM362, prevents liver failure and promotes liver regeneration after extensive hepatectomy. Surgery 143: 545-555, 2008.

33. Zinszner H, Kuroda M, Wang X, et al: CHOP is implicated in programmed cell death in response to impaired function of the endoplasmic reticulum. Genes Dev 12: 982-995, 1998.

34. Garcea G and Maddern GJ: Liver failure after major hepatic resection. J Hepatobiliary Pancreat Surg 16: 145-155, 2009.

35. Nakadaira K, Tsukada K, Sakaguchi T, et al: A pharmacological analysis of prostaglandin E1 on portal blood flow after partial hepatectomy in rats. Surg Today 23: 277-279, 1993.

36. Fausto $\mathrm{N}$ and Riehle KJ: Mechanisms of liver regeneration and their clinical implications. J Hepatobiliary Pancreat Surg 12: 181-189, 2005.

37. Selzner N, Selzner M, Odermatt B, Tian Y, Van Rooijen N and Clavien PA: ICAM-1 triggers liver regeneration through leukocyte recruitment and Kupffer cell-dependent release of TNF-alpha/IL-6 in mice. Gastroenterology 124: 692-700, 2003.

38. Cataldegirmen G, Zeng S, Feirt N, et al: RAGE limits regeneration after massive liver injury by coordinated suppression of TNF-alpha and NF-kappaB. J Exp Med 201: 473-484, 2005.

39. Selzner N, Selzner M, Tian Y, Kadry Z and Clavien PA: Cold ischemia decreases liver regeneration after partial liver transplantation in the rat: A TNF-alpha/IL-6-dependent mechanism. Hepatology 36: 812-818, 2002.

40. Morita T, Togo S, Kubota T, et al: Mechanism of postoperative liver failure after excessive hepatectomy investigated using a cDNA microarray. J Hepatobiliary Pancreat Surg 9: 352-359, 2002.

41. Malhi H, Guicciardi ME and Gores GJ: Hepatocyte death: a clear and present danger. Physiol Rev 90: 1165-1194, 2010.

42. Malhi H and Kaufman RJ: Endoplasmic reticulum stress in liver disease. J Hepatol 54: 795-809, 2011. 